\title{
ASPECTOS TECNOLÓGICOS E SENSORIAIS DO "IOGURTE" DE SOJA ENRIQUECIDO COM CÁLCIO'
}

\author{
Daniela Cardoso UMBELINO², Elizeu Antonio ROSS| ${ }^{3, *}$,
} Helena Maria André Bolini CARDELLO ${ }^{4}$, José Salvador LEPERA ${ }^{5}$

\begin{abstract}
RESUMO
O objetivo deste trabalho foi verificar a viabilidade de enriquecimento do "iogurte"de soja com cálcio, para que o produto apresentasse níveis deste mineral equivalentes ou superiores aos encontrados nos iogurtes tradicionais e mantivesse as propriedades tecnológicas e sensoriais adequadas. Foram testados cinco sais: carbonato, citrato, fosfato, gliconato e lactato de cálcio, na concentração de $600 \mathrm{mg}$ de cálcio elementar/L. Os produtos foram avaliados quanto ao tempo de fermentação, $\mathrm{pH}$, acidez titulável, viscosidade, consistência, concentração de cálcio e propriedades sensoriais (testes de diferença do controle e aceitação). A adição de cálcio ao "iogurte" de soja na forma de citrato, fosfato, carbonato, gliconato e lactato de cálcio, apesar de provocar alterações na acidez titulável, viscosidade e consistência, não conferiu propriedades sensoriais indesejáveis ao produto e o tempo de fermentação não excedeu aos verificados nos processos industriais convencionais. Desta forma todos os sais testados se mostraram viáveis para o processo de enriquecimento do "iogurte" de soja com cálcio.
\end{abstract}

Palavras-chave: "iogurte" de soja; enriquecimento; cálcio; análise sensorial.

\section{SUMMARY}

SENSORIAL AND TECHNOLOGICAL ASPECTS OF CALCIUM ENRICHMENT OF A SOY-WHEY-YOGURT. The aim of this work was to obtain a soy-whey-yogurt with the same or higher calcium levels than that of traditional ones and with suitable sensory and technological properties. Five salts were tested: calcium carbonate, citrate, phosphate, gliconate and lactate, in the concentration of $600 \mathrm{mg}$ of the elementary calcium/L.The fermentation time, $\mathrm{pH}$, titratable acidity, viscosity, consistency, iron concentration and sensory properties (difference from the control and acceptance tests) of the product were evaluated. The addition of calcium to the soy-whey-yogurt as carbonate, citrate, phosphate, gliconate and lactate, despite the changes in the titratable acidity, viscosity, consistency and fermentation time, causes no adverse effects in the sensory properties indicating that any calcium salts studied could be used in the soy-wheyyogurt enrichment process.

Keywords: soy-whey yogurt; enrichment; calcium; sensory analysis.

\footnotetext{
${ }_{1}^{1}$ Recebido para publicação em 28/10/99. Aceito para publicação em 09/08/01.

${ }^{2}$ Autora da Dissertação de Mestrado da qual este artigo faz parte. Faculdade de Engenharia de Alimentos - UNICAMP. C.P. 6121, CEP 13086-970, Campinas-SP.

${ }^{3}$ Faculdade de Ciências Farmacêuticas - UNESP. Caixa Postal 502, CEP 14801-902, Araraquara-SP.

${ }^{4}$ Faculdade de Engenharia de Alimentos - UNICAMP. C. P. 6121, CEP 13086-970, Campinas-SP.

${ }^{5}$ Depto. Princípios Ativos Naturais e Toxicológicos - FCF- UNESP.

C. P. 502, CEP 14801-902, Araraquara-SP.

${ }^{*}$ A quem a correspondência deve ser enviada.
}

\section{1 - INTRODUÇÃO}

O "iogurte" de soja é um produto fermentado obtido a partir do extrato aquoso de soja, de boa aceitabilidade e custo reduzido [12]. Este produto, apesar de manter preservadas as características nutricionais dos produtos fermentados tradicionais, difere significativamente destes em relação ao conteúdo mineral, sendo deficiente, principalmente em cálcio.

O consumo de quantidades adequadas de cálcio é fundamental para a obtenção de uma massa óssea ótima durante as duas primeiras décadas de vida, auxiliando na prevenção da osteoporose em adultos [3, 8, 9].

O teor de cálcio no "iogurte" de soja pode ser aumentado através da prática do enriquecimento $[5,17]$ Porém, a adição de cálcio ao "iogurte" de soja é particularmente difícil, pois as proteínas da soja são sensíveis e precipitam pela ação deste mineral. Entretanto, a estabilidade da proteína da soja pode ser aumentada pelo uso de um agente quelante adequado [11, 17, 19].

O objetivo deste trabalho foi verificar a viabilidade de enriquecimento do "iogurte" de soja com fontes adequadas de cálcio para que o produto apresente níveis deste mineral equivalentes ou superiores aos encontrados nos iogurtes tradicionais sem que isso provocasse alterações nas suas propriedades sensoriais e tecnológicas.

\section{2 - MATERIAL E MÉTODOS}

\section{1 - Material}

Amostras de "iogurte" de soja enriquecidas com $600 \mathrm{mg}$ de cálcio na forma de: citrato tribásico de cálcio (Montedison Farmacêutica), carbonato de cálcio (Merck), fosfato tribásico de cálcio (Merck), gliconato de cálcio (Merck) e lactato de cálcio (Purac).

\section{2 - Métodos}

\subsection{1 - Obtenção das amostras}

Foram processadas cinco amostras de "iogurte" à base de extrato aquoso de soja [12], enriquecidas com $600 \mathrm{mg}$ de cálcio elementar por litro, e uma amostra sem adição de cálcio que foi utilizada como padrão. As misturas foram inoculadas com $4 \%$ de um inóculo misto comercial (Christian Hansen Ind. e Com. Ltda), constituído de partes iguais de L.delbrueckii ssp. bulgaricus e $S$. thermophilus. O processo de fermentação foi considerado completo quando o pH da mistura atingiu 4,4-4,5. 


\subsection{2 - Análises físico-químicas}

- Determinação do tempo de fermentação

Correspondeu ao tempo total de incubação das amostras em estufa bacteriológica a $37^{\circ} \mathrm{C}$, até que $\mathrm{O}$ $\mathrm{pH}$ fosse reduzido a 4,4-4,5 [15].

- Determinação do $\mathrm{pH}$

Realizada em pHmetro digital Micronal, mod. 320, com eletrodo de vidro combinado.

- Determinação da acidez titulável

A acidez titulável foi determinada no final dos processos fermentativos, utilizando-se solução de $\mathrm{NaOH}$ $1 / 9 \mathrm{~N}$ e os resultados foram expressos em graus Dornic ( ${ }^{\circ}$ ) [7].

- Determinação de cálcio

Realizada por espectrofotometria de absorção atômica em espectrofotômetro Perkin Elmer modelo 3110, atomização em chama, após precipitação protéica com ácido tricloroacético a $20 \%$, seguida de centrifugação a 2000rpm, durante 20 minutos. A calibração foi construída por múltipla adição no intervalo de 0 a 2000mg/L, utilizando padrão Tritisol (Merck) $1000 \mu \mathrm{g} / \mathrm{mL}$. A ionização foi corrigida com adição de cloreto de lantânio na concentração final de $0,2 \%$. As condições instrumentais foram: lâmpada de cátodo oco para cálcio (Narva) sob corrente de 7,5mA, comprimento de onda de 422,7 com fenda de saída de $0,7 \mathrm{~mm}$. Todas as amostras foram analisadas em triplicata.

- Determinação de viscosidade

Medida pela leitura direta em viscosímetro rotacional da marca WAAKE mod. VT 02, utilizando alíquotas de $120,0 \mathrm{~mL}$, mantidas à temperatura de $10^{\circ} \mathrm{C}$ [13].

\section{- Determinação de consistência}

Medida através da distância percorrida em centímetros por amostras de $80 \mathrm{~mL}$ mantidas a $10^{\circ} \mathrm{C}$, em um intervalo de tempo de 10 segundos, em consistômetro de Bostwick [13].

\subsection{3 - Análise sensorial}

- Teste de diferença do controle

Foram utilizados trinta provadores não-treinados que avaliaram a diferença global entre cada amostra (processada com diferentes sais de cálcio) e a amostracontrole (sem adição de cálcio) de acordo com o teste de "Diferença do Controle" [10]. As amostras de iogurte enriquecidas com cálcio e a amostra-controle foram apresentadas codificadas com algarismos de três dígitos e servidas à temperatura de refrigeração, juntamente com uma amostra-controle identificada com a letra C. Foi solicitada aos provadores a atribuição de notas a cada amostra, segundo o grau de diferença global em relação ao controle, em ficha apropriada. Foram definidos como parâmetros de diferença global o sabor, a cor, o aroma, a aparência, a viscosidade, a textura e outros atributos que o provador considerasse relevantes. Os resultados foram expressos como média da diferença em relação ao controle.
- Teste de aceitação

Trinta provadores não-treinados realizaram as análises sensoriais de aceitação das amostras em relação a aroma, cor, sabor e impressão global, através de escala hedônica não-estruturada de nove centímetros, ancoradas à esquerda pelo termo "desgostei muitíssimo"e à direita pelo termo "gostei muitíssimo", sendo as amostras apresentadas em blocos completos casualizados, de forma monádica [14].

\subsection{4 - Análise estatística dos resultados}

As medidas de acidez titulável, $\mathrm{pH}$ final, concentração de cálcio, viscosidade e consistência foram analisadas através de análise de variância e teste de médias de Tukey. Os dados obtidos no teste de diferença do controle foram avaliados através de análise de variância e testes de médias de Dunnett e os do teste de aceitação através de análise de variância e testes de médias de Tukey.

\section{3 - RESULTADOS E DISCUSSÃO}

\section{1 - Análises físico-químicas}

A adição de $600 \mathrm{mg}$ de cálcio na forma de citrato, fosfato e carbonato não alterou a estabilidade do produto, mesmo quando submetido a aquecimento de $95^{\circ} \mathrm{C}$ por $5 \mathrm{~min}$, prática rotineira no processamento de iogurte. Por outro lado, as amostras enriquecidas com gliconato e lactato de cálcio precipitaram com a elevação da temperatura, necessitando da adição de citrato de sódio (agente quelante), que nas concentrações de 0,15 e $0,30 \%$, respectivamente, conferiu estabilidade térmica satisfatória aos produtos.

A Tabela 1 mostra o tempo requerido por cada uma das diferentes amostras para reduzir o $\mathrm{pH}$ a 4,4-4,5. O maior tempo de fermentação observado para as amostras contendo lactato e gliconato de cálcio reforça as observações de outros autores que afirmam que, normalmente, os sais de cálcio prolongam o processo fermentativo [5].

TABELA 1. Tempo de fermentação, $\mathrm{pH}$ final e acidez titulável de amostras enriquecidas com diferentes sais de cálcio.

\begin{tabular}{cccc}
\hline Amostras & $\begin{array}{c}\text { Tempo de } \\
\text { Fermentação }(\mathbf{h})\end{array}$ & $\begin{array}{c}\text { pH final } \\
(\mathbf{n}=\mathbf{3})\end{array}$ & $\begin{array}{c}\text { Acidez }\left({ }^{\circ} \mathbf{D}\right) \\
(\mathbf{n}=\mathbf{3})\end{array}$ \\
\hline Controle & $4: 00$ & $4,45 \pm 0,01^{\mathrm{a}}$ & $83 \pm 0,5^{\mathrm{d}}$ \\
Citrato & $5: 15$ & $4,42 \pm 0,01^{\mathrm{a}}$ & $86 \pm 0,0^{\mathrm{c}}$ \\
Fosfato & $5: 00$ & $4,44 \pm 0,01^{\mathrm{a}}$ & $82 \pm 0,0^{\mathrm{d}}$ \\
Carbonato & $5: 15$ & $4,44 \pm 0,01^{\mathrm{a}}$ & $86 \pm 0,5^{\mathrm{c}}$ \\
Gliconato* & $6: 00$ & $4,46 \pm 0,01^{\mathrm{a}}$ & $90 \pm 0,5^{\mathrm{a}}$ \\
& $6: 30$ & $4,47 \pm 0,01^{\mathrm{a}}$ & $88 \pm 0,0^{\mathrm{b}}$ \\
\hline Lactato** & & \\
\hline
\end{tabular}

* Adição de $0,15 \%$ de citrato de sódio (agente quelante) ** Adição de 0,30\% de citrato de sódio (agente quelante). num 
Segundo alguns pesquisadores [4, 18], esse efeito se deve à liberação de ácido glicônico e outros ácidos orgânicos, durante a produção de iogurtes enriquecidos com cálcio, os quais exercem efeito bacteriostático sobre o $S$. thermophilus e outras bactérias láticas. Do ponto de vista prático, pode-se afirmar que a elevação no tempo de fermentação observada neste estudo não inviabiliza o processo de enriquecimento, uma vez que 6:30h não excede o tempo habitual verificado nos processos industriais convencionais.

Ainda na Tabela 1, observa-se que, com exceção do fosfato de cálcio, os demais sais provocaram um aumento na acidez titulável dos produtos, em relação ao controle, embora os valores de $\mathrm{pH}$ tenham se mantido praticamente idênticos. Este efeito pode ser explicado, provavelmente, em razão do efeito do íon comum do principal ácido presente no produto, o ácido lático.

Em relação à avaliação reológica, cada amostra teve a viscosidade e a consistência determinadas e os resultados são apresentados na Tabela 2.

A amostra enriquecida com gliconato de cálcio apresentou menor viscosidade em relação às demais amostras, sendo a que mais se assemelhou ao controle (sem diferença significativa para $p<0,05)$. Foi, também, a amostra que apresentou o menor valor para consistência (escoamento mais rápido), sem diferir significativamente $(p<0,05)$ apenas das amostras enriquecidas com citrato e lactato de cálcio.

TABELA 2. Valores médios de viscosidade e consistência de amostras enriquecidas com diferentes sais de cálcio.

\begin{tabular}{ccc}
\hline $\begin{array}{c}\text { Amostras } \\
\text { Controle }\end{array}$ & $\begin{array}{c}\text { Viscosidade }(\mathbf{c P}) \\
(\mathbf{n}=\mathbf{3})\end{array}$ & $\begin{array}{c}\text { Consistência }(\mathbf{c m} / \mathbf{1 0 s}) \\
(\mathbf{n}=\mathbf{3})\end{array}$ \\
\hline Citrato & $3000 \pm 50^{\mathrm{c}}$ & $4,8 \pm 0,3^{\mathrm{b}}$ \\
Fosfato & $5000 \pm 50^{\mathrm{b}}$ & $4,3 \pm 0,3^{\mathrm{b}, \mathrm{b}}$ \\
Carbonato & $3433 \pm 58^{\mathrm{a}}$ & $4,8 \pm 0,3^{\mathrm{b}}$ \\
Gliconato & $2333 \pm 58^{\mathrm{c}}$ & $5,8 \pm 0,3^{\mathrm{a}}$ \\
& $2933 \pm 76^{\mathrm{b}}$ & $5,2 \pm 0,3^{\mathrm{a}, \mathrm{b}}$ \\
\hline
\end{tabular}

n= número de repetições

Médias com letras iguais na mesma coluna não diferem entre si $(p<0,05)$.

A Tabela 3 apresenta os níveis de cálcio determinados nas amostras de "iogurte" de soja controle e enriquecidas.

Observou-se que todas as amostras apresentaram níveis de cálcio superiores aos encontrados nos iogurtes tradicionais, que em média contêm 1200,00mg de cálcio/L [6]. Deve-se ressaltar ainda que, segundo a legislação brasileira, todos os produtos obtidos neste estudo, independentemente da fonte de cálcio utilizada, podem ser considerados enriquecidos, pois cada $100 \mathrm{~mL}$ dos "iogurtes"passaram a conter mais de $120 \mathrm{mg}$ de cálcio, valor que representa $15 \%$ da IDR de referência para todas as faixas etárias, exceto gestantes e lactantes [1, 2].

TABELA 3. Concentração de cálcio nas amostras enriquecidas com diferentes sais de cálcio.

\begin{tabular}{lc}
\hline $\begin{array}{c}\text { Amostras } \\
(\mathbf{n}=\mathbf{3})\end{array}$ & $\begin{array}{c}\text { Cálcio determinado }(\mathbf{m g} / \mathbf{L}) \\
\text { Controle }\end{array}$ \\
\hline Citrato & $1466 \pm 8^{\mathrm{a}}$ \\
Fosfato & $1439 \pm 7^{\mathrm{b}}$ \\
Carbonato & $1451 \pm 5^{\mathrm{a}, \mathrm{b}}$ \\
Gliconato & $1416 \pm 7^{\mathrm{c}}$ \\
Lactato & $1439 \pm 7^{\mathrm{c}}$ \\
\hline
\end{tabular}

\section{2 - Análise sensorial}

\subsection{1 - Teste de diferença do controle}

O teste de diferença do controle permitiu verificar se as amostras de "iogurte" de soja enriquecidas com $600 \mathrm{mg}$ de cálcio, na forma de citrato, fosfato, carbonato, gliconato e lactato, diferiam significativamente da amostra-controle (sem adição de cálcio) em termos globais.

Os resultados, avaliados através de análise de variância e teste de médias de Dunnett, demonstraram que apenas o "iogurte" enriquecido com gliconato de cálcio diferia significativamente do controle $(p<0,05)$. Esta diferença deve-se, provavelmente, ao elevado valor de acidez titulável $\left(90^{\circ} \mathrm{D}\right)$ e a baixa viscosidade $(2333 \mathrm{cP})$ apresentados pela amostra. As demais amostras estudadas não apresentaram diferença significativa quando comparadas com o controle (Tabela 4).

TABELA 4. Teste de diferença do controle das amostras enriquecidas com diferentes sais de cálcio.

\begin{tabular}{cc}
\hline Amostras & Médias \pm DP \\
\hline Controle & $3,13 \pm 2,45^{\text {a }}$ \\
Citrato de cálcio & $2,90 \pm 2,11^{\text {a }}$ \\
Fosfato de cálcio & $3,23 \pm 2,37^{\text {a }}$ \\
Carbonato de cálcio & $5,70 \pm 2,43^{\text {a }}$ \\
Gliconato de cálcio & \\
Lactato de cálcio & $4,43 \pm 2,76^{\text {b }}$
\end{tabular}

Médias com letras iguais na mesma coluna não diferem entre si $(p<0,05)$. 


\subsection{2 - Teste de aceitação}

O teste de aceitação avaliou os atributos de aroma, cor, sabor e impressão global dos "iogurtes" enriquecidos com cálcio e do "iogurte"-controle (sem adição de cálcio). Este teste foi realizado para verificar a resposta sensorial dos consumidores ou consumidores potenciais, em relação aos diferentes produtos obtidos.

Na Tabela 5 são apresentadas as médias dos atributos avaliados no teste de aceitação para as amostras utilizadas nesta etapa do estudo.

A análise de variância e teste de médias de Tukey mostrou que as amostras estudadas não apresentaram diferença significativa em relação ao aroma, cor sabor e impressão global, apesar da amostra-controle apresentar as maiores médias, em termos absolutos, em relação a todos os atributos avaliados.

Pode-se ainda observar que a amostra de "iogurte" de soja enriquecida com gliconato de cálcio, apesar de diferir em termos globais da amostra-controle, apresentou uma boa aceitação em relação a todos os atributos sensoriais avaliados, não diferindo significativamente das demais amostras.

TABELA 5. Teste de aceitação das amostras enriquecidas com diferentes sais de cálcio

\begin{tabular}{ccccc}
\hline Amostras & Aroma & Cor & Sabor & I. Global \\
\hline Controle & $6,13 \pm 1,56$ & $6,91 \pm 1,43$ & $5,80 \pm 1,80$ & $6,23 \pm 1,39$ \\
Citrato de cálcio & $5,36 \pm 1,84$ & $6,31 \pm 1,46$ & $5,27 \pm 1,90$ & $5,64 \pm 1,44$ \\
Fosfato de cálcio & $5,18 \pm 1,96$ & $6,22 \pm 1,59$ & $5,41 \pm 2,18$ & $5,52 \pm 1,85$ \\
Carbonato de cálcio & $5,68 \pm 1,80$ & $6,70 \pm 1,40$ & $5,63 \pm 1,70$ & $5,63 \pm 1,60$ \\
Gliconato de cálcio & $5,48 \pm 1,98$ & $5,87 \pm 2,02$ & $5,57 \pm 1,82$ & $5,76 \pm 1,85$ \\
Lactato de cálcio & $4,92 \pm 1,75$ & $5,98 \pm 1,62$ & $4,55 \pm 1,90$ & $5,05 \pm 1,7$ \\
DMS & 1,31 & 1,14 & 1,38 & 1,22 \\
\end{tabular}

DMS: Diferença mínima significativa obtida através do teste de médias de Tukey $(p<0,05)$.

Não foram observadas diferenças significativas $(p<0,05)$ entre todas as amostras.

Os resultados obtidos demonstraram que a adição de citrato, fosfato, carbonato, gliconato e lactato de cálcio não confere propriedades sensoriais indesejáveis ao "iogurte"de soja, tornando possível a utilização de qualquer um destes sais no processo de enriquecimento com cálcio.

Estes resultados estão em concordância com os observados na literatura. WEINGARTNER, NELSON, ERDMAN [16] conduziram um estudo para verificar a possibilidade de utilização de citrato e fosfato de cálcio para o enriquecimento de "leite"de soja. Observaram, através do teste triangular, que a amostra enriquecida com citrato de cálcio não diferia significativamente da amostra não-enriquecida. Em um outro teste sensorial aplicado, as amostras enriquecidas com $30 \mathrm{mM}$ de uma mistura de citrato e fosfato de cálcio apresentaram melhor sabor, quando comparadas com a amostra sem adição de cálcio, indicando que a adição desses sais, isolados ou em associação, não alterava adversamente as propriedades sensoriais do "leite"de soja.

\section{4 - CONCLUSÕES}

Com base nos resultados obtidos, é possível concluir que a adição de cálcio ao "iogurte" de soja na forma de citrato, fosfato, carbonato, gliconato e lactato de cálcio, apesar de provocar alterações na acidez titulável, viscosidade e consistência, não conferiu propriedades sensoriais indesejáveis ao produto e o tempo de fermentação não excedeu aos verificados nos processos industriais convencionais. Desta forma, todos os sais testados podem ser empregados com sucesso no processo de enriquecimento do "iogurte" de soja.

\section{5 - REFERÊNCIAS BIBLIOGRÁFICAS}

[1] BRASIL. Ministério da Saúde. Portaria n 31, 13 jan. 1998, Regulamento técnico para fixação de identidade e qualidade de alimentos adicionados de nutrientes essenciais. Diário Oficial da União, Brasília, 1998.

[2] BRASIL. Ministério da Saúde. Portaria n 33, 13 jan. 1998, Ingestão diária recomendada (IDR) para proteínas, vitaminas e minerais. Diário Oficial da União, Brasília, 1998.

[3] DUTRA de OLIVEIRA, J.E., MARCHINI, J.S. Ciências nutricionais. São Paulo: Sarvier, 1998. 403p.

[4] EISHENAWY, K., MARTH, E.H. Behavior of Listeria monocytogenes in the presence of gluconic acid and during preparation of cottage cheese curd using gliconic acid. J. Dairy Sci., v. 73, p. 1429, 1990.

[5] FLIGNER, K., LINDAMOOD, J.B., HANSEN, P.M.T. Fortification of low-fat plain yogurt with calcium gluconate. Cult. Dairy Prod. J. v. 23, p. 5-9, 1988.

[6] FRANCO, G. Tabela de Composição dos Alimentos. 9.ed. São Paulo: Atheneu, 1996, p. 232-7.

[7] INSTITUTO ADOLFO LUTZ. Normas Analíticas do Instituto Adolfo Lutz: métodos químicos e físicos para análise de alimentos. 2ed. São Paulo, 1976. v. 1, 371p.

[8] MATCKOVIC, V. FONTANA, D., TOMINAC, C., GOEL, P., CHESNUT, C. Factors that influence peak bone mass formation: a study of calcium balance and the inheritance of bono mass in adolescent females. Am. J. Clin. Nutr., v. 52, p. 878-88, 1990.

[9] MATCKOVIC, V. Calcium intake and peak bone mass. N. Engl. J. Med. v. 327, p. 119-120, 1992.

[10] MEILGARD, M., CIVILLE, G.V., CARR, T.B. Sensory avaliation techniques. Boca Ranton, Flórida: CRC Press, 1988. 281p.

[11] RASYID, F., HANSEN, P.M.T. Stabilization of soy milk fortified with calcium gluconate. Food Hydrocolloids., v. 4, p. 41522, 1991.

[12] ROSSI, E.A., REDDY, K.V., SILVA, R.S.S.F. Formulation of soy-whey yogurt, using response surface methodology. Arq. Biol. Tecnol., v. 27, p. 387-90, 1984.

[13] ROSSI, E.A, FARIA, J.B., BORSATO, D., BALDOCHI, F.L. Otimização de um sistema estabilizante para o "iogurte" de soja. Alim. Nutr., v. 2, p. 83-92, 1990.

[14] STONE, H., SIDEL, J.L. Sensory evaluation pratices. 2ed. London: Academic Press, 1993. 338p.

[15] TAMIME, A.Y., ROBINSON, R.K. Yoghurt: science and technology. Oxford: Pergamon, 1985, 431p. 
[16] WEINGARTNER, K.E., NELSON, A.I., ERDMAN, J.W. Effects of calcium addition on stability and sensory properties of soy beverage. J. Food Sci, v. 43, p. 256-7, 263, 1983.

[17] YAZICI, F., ALVAREZ, V.B., HANSEN, P.M.T. Fermentation and properties of calcium-fortified soy milk. J. Food Sci, v. 62, p. 457-61, 1997.
[18] YOUSEF, A.E., RUSLI, M. Modification of starter culture for production of calcium fortified yogurt. Cult. Dairy Prod.J., v. 30, p. 20-5, 1995.

[19] ZEMEL, M.B., SHELEF, L.A. Calcium fortified soy milk. European Patent Application. 0195167. p. 1-18, 1986.

\section{6 - AGRADECIMENTOS}

À CAPES pela concessão de bolsa de estudo. 